\title{
Morphology linked to miscibility in highly amorphous semi-conducting polymer/fullerene blends
}

John R. Tumbleston ${ }^{a}$, Liqiang Yang ${ }^{b}$, Wei You ${ }^{b}$, and Harald Ade ${ }^{a *}$

a. Department of Physics, North Carolina State University, Raleigh, North Carolina 27695

b. Department of Chemistry, University of North Carolina at Chapel Hill, Chapel Hill, North

Carolina 27599

*Email: hwade@ncsu.edu

TOC GRAPHICS

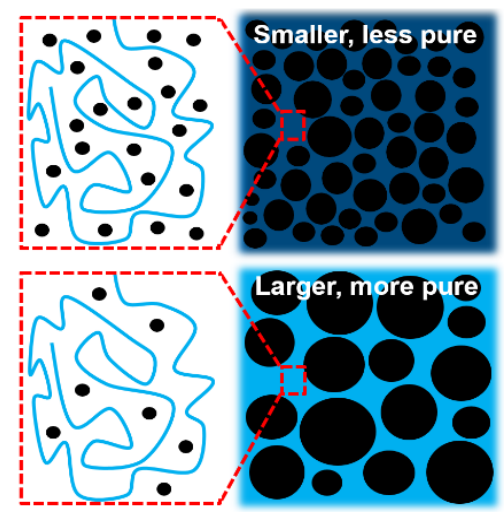


Abstract: Molecular miscibility is a defining property of mixtures of electron-donating polymers and electron-accepting fullerenes used in the photoactive layer of bulk heterojunction (BHJ) organic solar cells. Even though miscibility of fullerene in the polymer is a commonly observed property, quantitative measurements have unknown connections with other morphological properties such as domain size and domain purity. By varying the amount of fullerene loading in $\mathrm{BHJ}$ thin films, we show that morphological properties are related to miscibility via straightforward relationships. In particular, miscibility influences the sensitivity of domain spacing to fullerene loading and determines the domain volume fractions and the residual fullerene content in the mixed polymer-rich phase in devices. Not only does this work show the relationships between miscibility and morphology, but it also highlights the general importance in determining miscibility levels in BHJ systems in order to target optimum morphologies and domain purities.

Keywords: organic solar cells, X-ray scattering, bulk heterojunction

\section{Introduction}

Partial molecular miscibility of polymer and fullerene in bulk heterojunction (BHJ) solar cells has become a widely observed property of the polymer:fullerene blend (i.e., the active layer of BHJ solar cell) since its discovery a few years ago.[1-3] This had led to the inclusion of this important characteristic in the morphological description and paradigm of BHJ thin films along with its consideration in device performance and morphology formation.[4-9] For instance, the concept of domain purity has become an important morphological parameter since electrondonating and electron-accepting domains are not completely pure and are now known to contain some level of molecular mixing.[10-13] 
Even though partial miscibility has been observed in many BHJ systems,[14-16] there has only been limited work to relate miscibility levels to other defining properties of the morphology such as domain size and domain purity.[4, 5] In particular, it remains unclear how different miscibility limits will drive the establishment of domain size or purity during morphological formation. Miscibility should also influence the optimum ratio of electron donor to electron acceptor in BHJ devices because high miscibility will potentially lead to domains that are more likely to remain mixed. Answers to these important questions will help establish practical relationships between the fundamental property of molecular miscibility and performance-relevant morphological parameters.

In this Article, we show how intrinsic material miscibility between polymer and fullerene affects important characteristics of the morphology in straightforward ways by varying the ratio of electron-donating polymers to electron-accepting fullerenes. We find that higher miscibility causes domain spacing to be less sensitive to fullerene loading. We also show that with higher miscibility, more fullerene is needed to overcome the miscibility threshold before fullerene-rich domains can be formed. Furthermore, the scattering intensity from resonant soft X-ray scattering (R-SoXS) is maximized for greater PCBM loading for more highly miscible systems because a higher level of fullerene remains dissolved in the polymer network. Overall, this work highlights the importance of miscibility in affecting aspects of the morphology in polymer/fullerene BHJ thin films.

\section{Experimental Methods}

The polymer used in this work, PNDT-DTBT was synthesized according to previously published methods[17] and resulted in the following properties $\left(\mathrm{M}_{\mathrm{n}}\right.$ in $\mathrm{kg} / \mathrm{mol}$; polydispersity): hydrogen- 
based $(7.62 ; 2.13)$, fluorinated $(10.5 ; 2.70)$. Mixtures with varying loading of the fullerene, PCBM, and $10 \mathrm{mg} / \mathrm{mL}$ concentration for PNDT-DTBT were dissolved and cast from hot $\mathrm{CB}$ at $120{ }^{\circ} \mathrm{C}$ onto PEDOT:PSS-coated Si substrates in a nitrogen glovebox at $700 \mathrm{RPM}$ and dried at room temperature in a sealed petri dish for $12 \mathrm{hrs}$. STXM was conducted at beamline 5.3.2.2 of the ALS[18] using films floated onto TEM grids. GIWAXS was carried out at beamline 7.3.3 of the ALS[19] of films on the PEDOT:PSS-coated Si substrates using an incident angle of $\sim 0.12^{\circ}$. RSoXS measurements were conducted at beamline 11.0.1.2 of the ALS[20] by floating sections of blend films onto silicon nitride windows. R-SoXS scattering intensity was corrected for differences in film thickness as measured with profilometry. Composition variations were calculated from scattering at $282.5 \mathrm{eV}$ and $284.1 \mathrm{eV}$ photon energies. Densities of $1.1 \mathrm{~g} / \mathrm{cm}^{3}$ and $1.3 \mathrm{~g} / \mathrm{cm}^{3}$ were used for calculation of scattering volume for polymer and fullerene, respectively.

\section{Results and Discussion}

Figure 1a shows the chemical structure of the electron donating polymer, PNDT-DTBT, and the electron acceptor, PCBM (a soluble fullerene derivative), used in this work. Two variations in the polymer are employed, one with two fluorine atoms on the DTBT part of the polymer and another with the usual hydrogen atoms.[17] Fluorination is an ideal way to monitor how miscibility influences morphology since this chemical change constitutes a relatively minor difference between polymers where the conjugated aromatic units and side chains remain unchanged. The molecular weight is also similar although not identical between the fluorinated and hydrogen-based polymer (see Experimental for details and discussed below). All blends are processed from chlorobenzene (CB), which is an ideal processing solvent for this study since it leaves the polymer relatively amorphous making crystallinity differences minimal in comparisons and analyses. There was also no thermal treatment of the films. In general, the 
binodal phase boundary between the one phase and two phase regions of phase diagram depends on both the molecular weight and enthalpic properties of the materials used. Measuring the partial miscibility determines the binodal and thus captures the impact of both parameters.

Miscibility measurements of PCBM in non-fluorinated and fluorinated PNDT-DTBT using scanning transmission X-ray microscopy (STXM) reveal that the combined effect of fluorination and molecular weight causes the miscibility to be reduced by a factor of two for PNDT-DTBT from $26 \%$ to $13 \%$. STXM measurements were conducted at beamline 5.3.2.2 at the Advanced Light Source (ALS)[18] on CB-cast films that were solvent annealed to equilibrium prior to measurement and analyzed following previously established protocols.[21] The reduction in miscibility with fluorination has been observed in other blends[4] and is potentially a general effect due to enhanced repulsion of fullerene molecules in the presence of fluorine. 

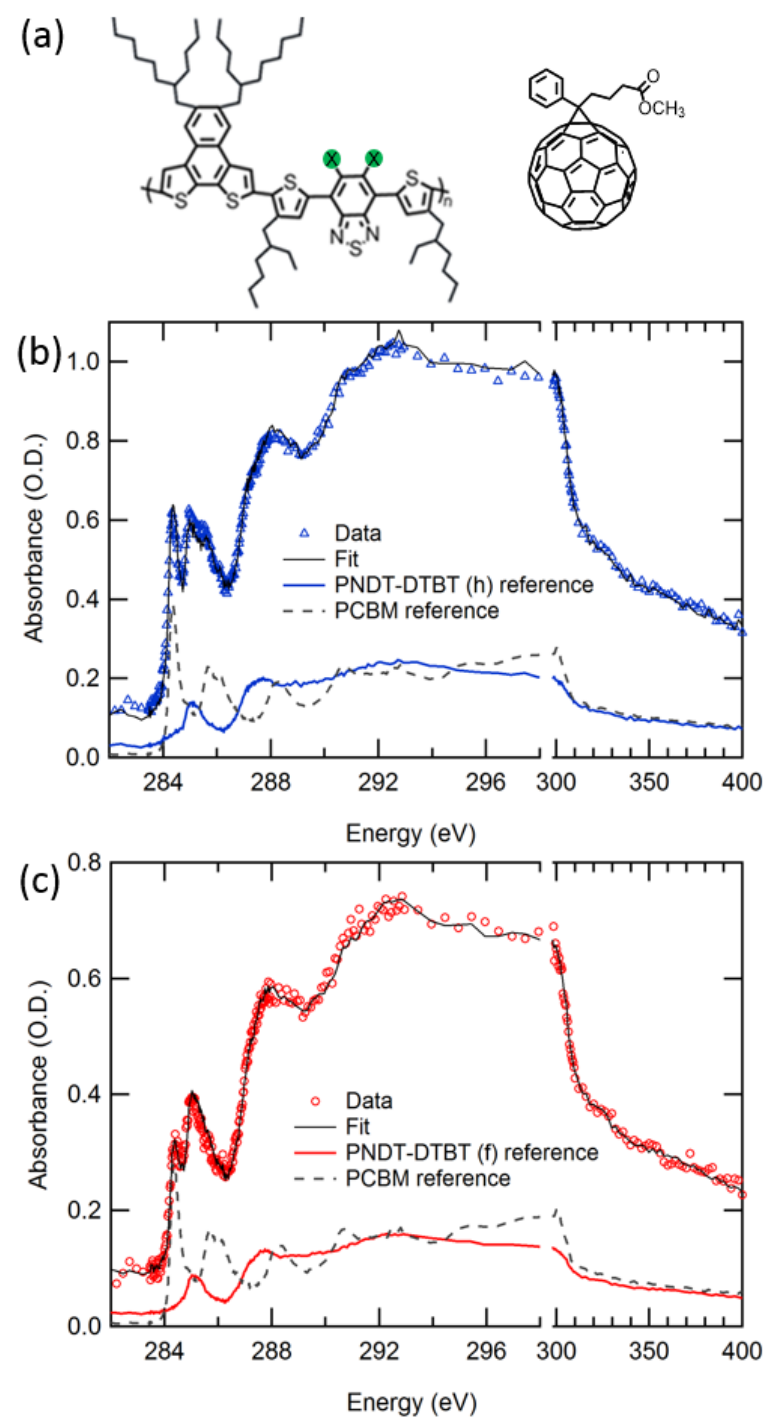

Figure 1. Molecular miscibility. (a) Chemical structures of PNDT-DTBT polymer and PCBM fullerene used in this study. The green circles marked with an " $\mathrm{X}$ " on the DTBT unit of the polymer are either hydrogen or fluorine. Scanning transmission X-ray microscopy scans of (a) hydrogenbased and (b) fluorinated blends that have been solvent annealed to equilibrium. Miscibility values of fullerene in polymer of $26 \%$ and $13 \%$, respectively, are determined from linear combination fits of the neat reference materials (also shown). 
While STXM miscibility measurements are an excellent quantitative tool to determine miscibility near equilibrium, the films are not device-relevant due to the prolonged solvent anneal required to reach equilibrium. This measurement is also not influenced by PCBM loading since additional PCBM from the increased fullerene loading will be expelled to PCBM crystals surrounding the depleted polymer matrix and will not affect the measured miscibility.[1,2] To assess the impact of miscibility in films with varying $\mathrm{D} / \mathrm{A}$ ratio, we utilized grazing incidence wide angle X-ray scattering (GIWAXS) to monitor the scattering signal from agglomerated fullerene[10] at beamline 7.3.3 of the ALS.[19] Figure 2a shows circularly averaged intensity data from 2-dimensional (2-D) scattering data along with multipeak fits. Four peaks with varying intensity are observed in the data, two corresponding to the polymer ordering and two corresponding to the fullerene ordering. Specifically, the peak at $q=0.3 \AA^{-1}$ corresponds to lamellar ordering of the polymer while the weaker peak at $1.7 \AA^{-1}$ is due to $\pi-\pi$ stacking of the polymer. The other two peaks, one at $0.7 \AA^{-1}$ and one at $1.4 \AA^{-1}$ represent fullerene aggregation. While others have monitored the more intense higher-q peak,[22] we utilize the lower-q feature at $0.7 \AA^{-1}$ to measure the extent of fullerene agglomeration in the thin films following previous work, as this avoids overlap with a diffuse scattering background from the polymer or substrate.[10]

Figure $2 \mathrm{~b}$ shows that the scattering intensity of the $0.7 \AA^{-1}$ peak increases with greater PCBM loading. Importantly, we find that the scattering intensity is higher for blends with the fluorinated polymer for equivalent PCBM loading, indicating that PCBM is more readily expelled from the polymer matrix to form agglomerated fullerene. This is consistent with the lower measured miscibility from the STXM analysis in Figure 1. In fact, linear fits have x-axis intercepts of $17 \%$ and $28 \%$ PCBM loading for fluorine and hydrogen-based blends, respectively. 
These intercepts correspond to the threshold concentration levels where PCBM is completely dissolved in the polymer matrix and are in good agreement from STXM analysis. Please also note that the scattering from the polymer is weak and void of any higher order peaks, indicating that the polymer is highly amorphous compared to semicrystalline polymers like P3HT[14] and when processing these same materials from a higher boiling point solvent such as dichlorobenzene.[17] Simple analysis of the (100) peak widths yields a maximum crystal size of only a few polymer chains. While the tight packing of these chains would exclude PCBM from being miscible in these regions, we surmise that polymer crystals constitute a very low volume fraction in all blends. This removes the complication of significant polymer crystallinity, which can be an important driver of morphological evolution if present.[14, 23, 24] 


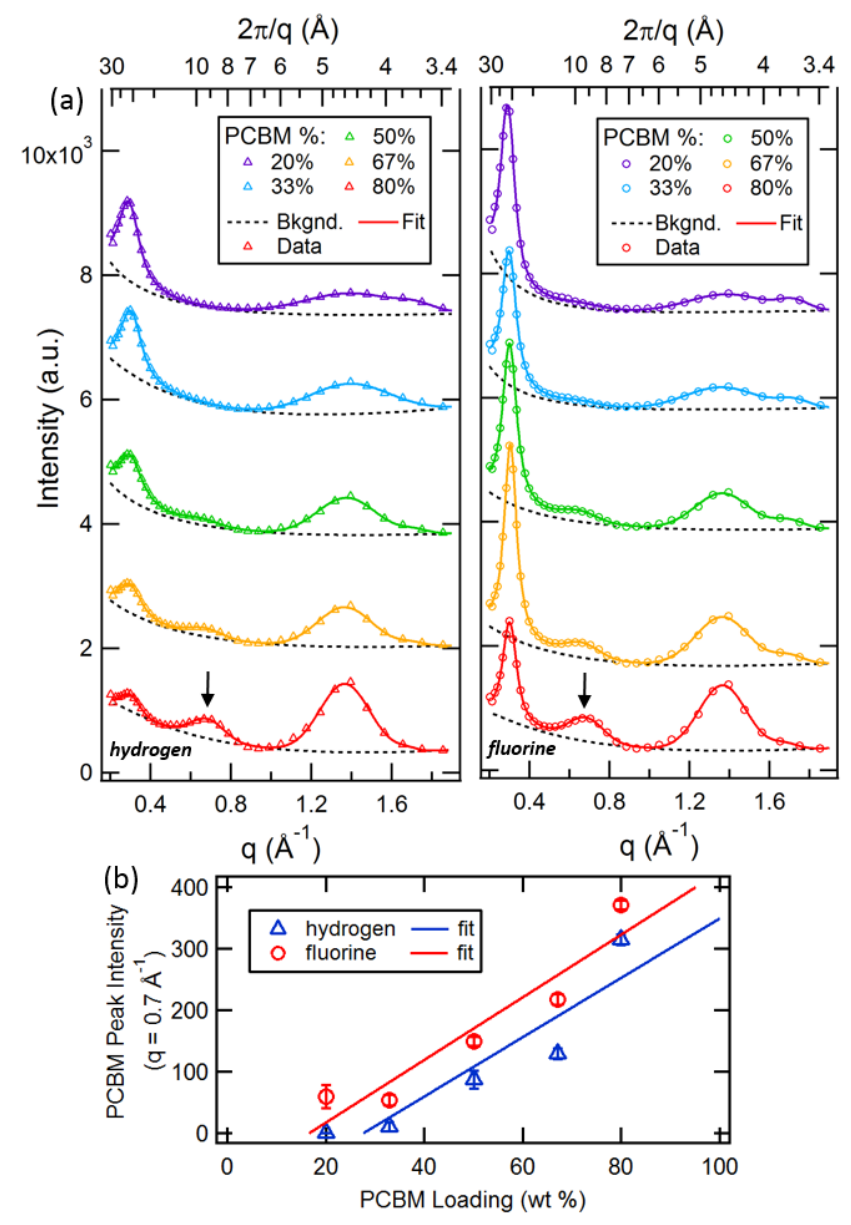

Figure 2. GIWAXS. (a) Circular averages of 2-dimensional grazing incidence wide angle X-ray scattering data of hydrogen-based (left) and fluorinated (right) blends with different amounts of PCBM loading. The data are fit with four peaks along with a background (also shown). The peak at $0.7 \AA^{-1}$ is indicative of fullerene agglomeration with the fitted peak intensities shown in (b) for the ten different samples. X-axis intercepts of linear fits of $17 \mathrm{wt} \%$ and $28 \mathrm{wt} \% \mathrm{PCBM}$ concentration are in agreement with values determined from STXM miscibility measurements in Figure 1. The model corresponding to the linear fit assumes that all PCBM above the miscibility threshold is not dissolved in the polymer and is leading to aggregation that can be measured by WAXS. 
Now that the miscibility has been fully assessed, we move to focus on the link between miscibility and morphology. Figure 3 shows circularly integrated intensity profiles of 2-D resonant soft X-ray scattering (R-SoXS) measurements at beamline 11.0.1.2 of the ALS[20] on blend films with variable PCBM loading. Circular integration corresponds to Lorentz correction as labeled in the figures. Data were acquired at a photon energy of $284.1 \mathrm{eV}$ where the contrast between polymer and fullerene is high for these materials, yet does not lead to beam damage or background fluorescence.[17] The peak in the Lorentz-corrected data is known as the long period $[9,25]$ and is representative of the spacing between centers of like domains. We observe that the scattering peak moves to higher scattering angle (i.e., smaller spacing) as PCBM loading is reduced. This occurs for blends of both polymer types, but the scattering peak consistently occurs at larger spacing for blends with the fluorinated polymer. Domain spacing values are shown in Figure 4a as a function of PCBM loading. The scattering data in Figure 3 is also shaped similarly, which indicates a similar distribution of spatial frequencies in all samples (except for those with low fullerene loading where scattering intensity is weak). An increase in domain spacing with greater fullerene loading has been qualitatively noted for other blends of amorphous polymer with PCBM.[26-28] We now show that the miscibility level is related to the sensitivity of domain spacing to PCBM loading with lower miscibility (e.g. fluorinated blend) resulting in greater sensitivity. This is due to the greater propensity for fullerene to be excluded from the polymer-rich phase during morphology formation and corresponds to a deeper quench into the two phase region. It is interesting that the effect of the larger driving force from the deeper quench is dominant and overcomes the effect of molecular weight which should lead to smaller domains for the fluorinated polymer.[29] 

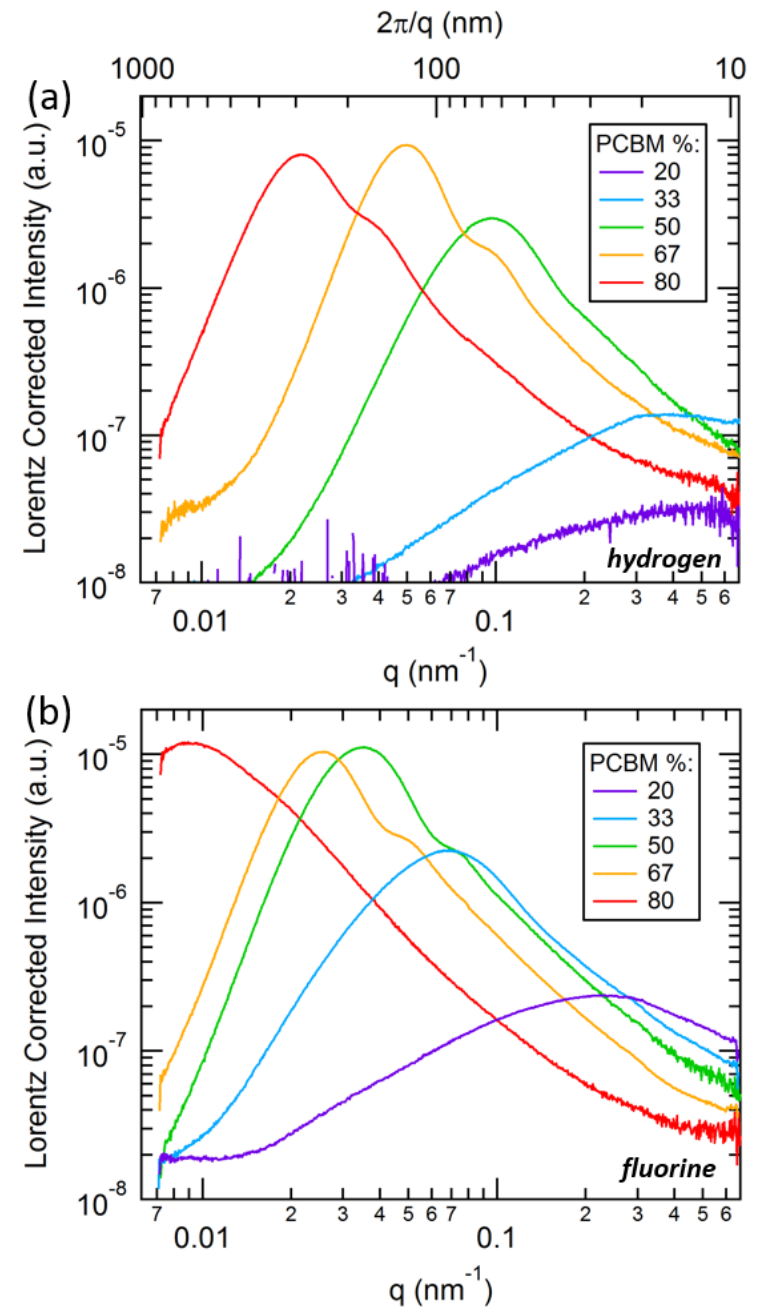

Figure 3. $R$-SoXS. Lorentz corrected resonant soft X-ray scattering data for $284.1 \mathrm{eV}$ photon energy for (a) hydrogen-based and (b) fluorinated blends with variable PCBM loading. The peaks in the scattering data are representative of domain spacing between centers of like domains while integration of the total scattering signal is related to domain purity in the samples.

Along with quantifying the domain spacing, the total scattering intensity (TSI) of RSoXS also provides the average composition variations between polymer-rich and fullerene-rich domains in the samples. $[4,5]$ TSI is given by: 
$I_{t o t}=\int_{0}^{\infty} I(q) q^{2} d q=2 \pi^{2} V \varphi(1-\varphi) \Delta n^{2}$

where $I(q)$ is the scattering intensity at scattering vector $q, V$ is the scattering volume and includes the film thickness, $\varphi$ is the volume fraction of one phase, and $\Delta n^{2}$ is the domain contrast. The optical contrast, $\Delta n^{2}$, quantifies the level of mixing between domains and is a measure of relative domain purity in a 2-phase system. TSI is experimentally determined by integration of the Lorentz-corrected scattering data in Figure 3 and is shown in Figure 4b. Peak values occur for $50 \%$ and $67 \%$ PCBM loading for fluorine and hydrogen-based blends, respectively.

Since scattering arises from the difference in optical properties between domains, the TSI is proportional to the product of the volume fraction of each phase, $\varphi(1-\varphi)$, as shown in equation $1 .[5,23]$ When including the volume fraction, $m$, that corresponds to the miscibility limit from STXM, and expressing $\varphi(1-\varphi)$ in terms of the PCBM loading of the mixture used, it can be written as:

$$
V=(1+m)(1-[P C B M])([P C B M]-m)
$$

where $[P C B M]$ is derived from the nominal PCBM loading and would be the volume fraction of PCBM in a completely phase separated mixture assuming nominal PCBM and polymer densities. The factor $(1+m)$ describes the swelling of the polymer-rich domains due to the dispersed PCBM, which otherwise would have a volume fraction $(1-[P C B M])$. The factor, $([P C B M]-m)$, is the PCBM volume left after losing $m$ to the polymer-rich domains. The volume fraction factors are overplotted with the TSI in Figure $4 \mathrm{~b}$ for hydrogen and fluorine-based blends using the measured miscibilities from Figure 1. Higher miscibility shifts the peak to higher PCBM loading and also reduces the scattering intensity for all PCBM loadings. By definition, 
the $\mathrm{x}$ intercepts are the miscibility limits from STXM, values that might be slightly too high relative to the R-SoXS data. Still, this simple model adequately reproduces both the lower intensities for the higher miscibility hydrogen-based blend and also the shift in peak TSI to higher PCBM loading. Knowing the miscibility between polymer and fullerene, a target loading of PCBM in the solution can be easily determined that will maximize the scattering intensity using equation 1.

Importantly, the trend in TSI is consistent with the miscibility described above. The hydrogen-based blend has overall lower scattering. The good agreement between TSI and our simple model indicates that the morphologies observed have similar relative scattering contrast, i.e. $\Delta n^{2}$, and thus purities. We therefore infer to have more mixed polymer-rich domains for hydrogen-based blends at all PCBM loading owing to the higher molecular miscibility of fullerene with polymer. On the other hand, the lower miscibility of the fluorine-based blend leads to greater expulsion of PCBM from the polymer matrix into PCBM-rich domains and thus results in higher domain purity. These conclusions from R-SoXS are consistent with the GIWAXS results that showed higher signal from PCBM aggregation for fluorine based blends for all PCBM loadings above the miscibility limit. 

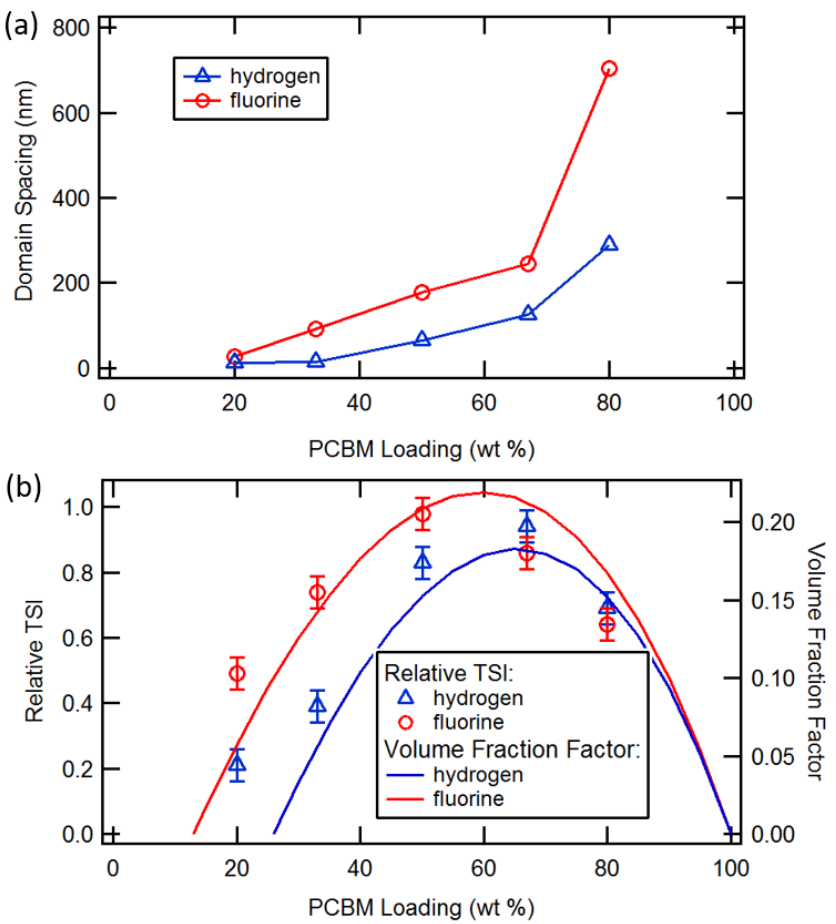

Figure 4. Morphological parameters. (a) Domain spacing (i.e. long periods) derived from scattering peaks in Figure 3. (b) Relative total scattering intensity (TSI) as function of PCBM loading for both hydrogen and fluorine-based PNDT-DTBT blends. Overplots of modeled volume fraction factors using miscibility values from Figure 1 correctly estimates the overall shape and peak PCBM loadings in TSI for both blends. Since the scattering volume dominates the relative TSI, domain purities are insensitive to PCBM loading and are higher for fluorinated blends due to the lower miscibility of PCBM in polymer. Error bars were determined from differences in total scattering intensities for different X-ray scattering energies and uncertainties in film thicknesses.

In terms of device performance, fullerene loading has long been known to be an important parameter to optimize.[30-33] Along with strongly affecting light absorption, the extent of fullerene agglomeration can also impact energetics and charge separation.[23, 34-36] Even though showing relationships with performance is outside the scope of this work due to the complexity of accurately correcting for optical absorption differences as PCBM loading changes, 
this work does demonstrate that there is a trade-off between domain size and purity in highly amorphous blends. Traditionally, small spacing on the order of the exciton diffusion length and high domain purity are desired simultaneously for high performance. This work shows that high purity levels via lower miscibility can be achieved but at the expense of larger domain spacing when the materials are highly amorphous. The extent of this trade-off depends on the fundamental miscibility between polymer and fullerene.

\section{Conclusion}

In conclusion, this work provides a first step towards linking the fundamental miscibility levels with measured morphological parameters critical to device performance. Not only are there systematic trends in both domain spacing and domain purity, but the difference in miscibility effectively predicts differences in domain volume fraction. At least for systems comprised of amorphous polymers, reducing PCBM loading will lead to smaller domain spacing.

Overall, our study indicates that PCBM loading is a critical parameter to optimize, because it influences the morphology in ways that can now be anticipated from molecular miscibility. This work illustrates that achieving at the same time small domains for good short circuit current (which is easier achieved with highly miscible systems) and pure domains for good fill factor (which is easier achieved with more immiscible systems), is likely to remain a considerable device fabrication challenge. These opposing forces might be one of the reasons why solvent additives are often needed to control the morphology in these devices in order to maximize the device efficiency. Fullerene miscibility might thus be a factor, alongside HOMO-LUMO level control and solubility in organic solvents, which needs to be considered when synthesizing new materials. 
Acknowledgment: X-ray characterization and analysis by JRT and HA was supported by DOE, OS, BES, MSE (DE-FG02-98ER45737). X-ray data were acquired at the ALS, which is supported by DOE (DE-AC02-05CH1123). LY and WY are supported by the Office of Naval Research (N000141110235) and an NSF CAREER Award (DMR-0954280). WY is a Camille Dreyfus Teacher-Scholar. The authors thank David Kilcoyne at ALS beamline 5.3.2.2, Alexander Hexemer and Steven Alvarez at beamline 7.3.3, and Cheng Wang and Anthony Young at beamline 11.0.1.2 for assistance with data acquisition and helpful discussions.

\section{References:}

[1] B. Watts, W.J. Belcher, L. Thomsen, H. Ade, P.C. Dastoor, Macromolecules, 42 (2009) 8392-8397.

[2] B.A. Collins, E. Gann, L. Guignard, X. He, C.R. McNeill, H. Ade, J. Phys. Chem. Lett., 1 (2010) 3160-3166.

[3] N.D. Treat, M.A. Brady, G. Smith, M.F. Toney, E.J. Kramer, C.J. Hawker, M.L. Chabinyc, Adv. Energy Mater., 1 (2011) 82-89.

[4] A.C. Stuart, J.R. Tumbleston, H. Zhou, W. Li, S. Liu, H. Ade, W. You, J. Am. Chem. Soc., 135 (2013) 1806-1815.

[5] B.A. Collins, Z. Li, J.R. Tumbleston, E. Gann, C.R. McNeill, H. Ade, Adv. Energy Mater., 3 (2013) 65-74.

[6] N.D. Treat, A. Varotto, C.J. Takacs, N. Batara, M. Al-Hashimi, M.J. Heeney, A.J. Heeger, F. Wudl, C.J. Hawker, M.L. Chabinyc, J. Am. Chem. Soc., 134 (2012) 15869-15879.

[7] J.R. Tumbleston, A.C. Stuart, E. Gann, H. Yan, B.A. Collins, W. You, H. Ade, Adv. Funct. Mater., 23 (2013) 3463-3470. 
[8] D. Chen, F. Liu, C. Wang, A. Nakahara, T.P. Russell, Nano Lett., 11 (2011) 2071-2078.

[9] W. Yin, M. Dadmun, ACS Nano, 5 (2011) 4756-4768.

[10] J.A. Bartelt, Z.M. Beiley, E.T. Hoke, W.R. Mateker, J.D. Douglas, B.A. Collins, J.R.

Tumbleston, K.R. Graham, A. Amassian, H. Ade, J.M.J. Fréchet, M.F. Toney, M.D. McGehee, Adv. Energy Mater., 3 (2012) 364-374.

[11] J.D. Roehling, K.J. Batenburg, F.B. Swain, A.J. Moulé, I. Arslan, Adv. Funct. Mater., 23 (2013) 2115-2122.

[12] M. Pfannmöller, H. Flügge, G. Benner, I. Wacker, C. Sommer, M. Hanselmann, S. Schmale, H. Schmidt, F.A. Hamprecht, T. Rabe, W. Kowalsky, R.R. Schröder, Nano Lett., 11 (2011) 3099-3107.

[13] W. Ma, J.R. Tumbleston, M. Wang, E. Gann, F. Huang, H. Ade, Adv. Energy Mater., 3 (2013) 864-872.

[14] B.A. Collins, J.R. Tumbleston, H. Ade, J. Phys. Chem. Lett., 2 (2011) 3135-3145.

[15] F. Liu, Y. Gu, J.W. Jung, W.H. Jo, T.P. Russell, J. Polym. Sci. Part B, 50 (2012) 1018-1044.

[16] C.R. McNeill, H. Ade, J. Mater. Chem. C, 1 (2013) 187 - 201.

[17] L. Yang, J.R. Tumbleston, H. Zhou, H. Ade, W. You, Energy Environ. Sci., 6 (2013) 316326.

[18] A.L.D. Kilcoyne, T. Tyliszczak, W.F. Steele, S. Fakra, P. Hitchcock, K. Franck, E. Anderson, B. Harteneck, E.G. Rightor, G.E. Mitchell, A.P. Hitchcock, L. Yang, T. Warwick, H. Ade, J. Synchrotron Radiat., 10 (2003) 125-136.

[19] A. Hexemer, W. Bras, J. Glossinger, E. Schaible, E. Gann, R. Kirian, A. MacDowell, M. Church, B. Rude, H. Padmore, J. Phys. Conf. Ser., 247 (2010) 012007. 
[20] E. Gann, A. Young, B.A. Collins, H. Yan, J. Nasiatka, H.A. Padmore, H. Ade, A. Hexemer, C. Wang, Rev. Sci. Instrum., 83 (2012) 045110.

[21] B.A. Collins, H. Ade, J. Electron. Spectrosc. Relat. Phenom., 185 (2012) 119-128.

[22] A.J. Pearson, T. Wang, A.D.F. Dunbar, H. Yi, D.C. Watters, D.M. Coles, P.A. Staniec, A. Iraqi, R.A.L. Jones, D.G. Lidzey, Adv. Funct. Mater., 24 (2014) 659-667.

[23] S.V. Kesava, Z. Fei, A.D. Rimshaw, C. Wang, A. Hexemer, J.B. Asbury, M. Heeney, E.D. Gomez, Adv. Energy Mater., (2014) DOI: 10.1002/aenm.201400116.

[24] M. Campoy-Quiles, T. Ferenczi, T. Agostinelli, P.G. Etchegoin, Y. Kim, T.D. Anthopoulos, P.N. Stavrinou, D.D.C. Bradley, J. Nelson, Nat. Mater., 7 (2008) 158-164.

[25] N. Stribeck, X-Ray Scattering of Soft Matter Springer, Berlin, Berlin, 2007.

[26] H. Hoppe, M. Niggemann, C. Winder, J. Kraut, R. Hiesgen, A. Hinsch, D. Meissner, N.S. Sariciftci, Adv. Funct. Mater., 14 (2004) 1005-1011.

[27] D. Veldman, O. İpek, S.C.J. Meskers, J.r. Sweelssen, M.M. Koetse, S.C. Veenstra, J.M.

Kroon, S.S.v. Bavel, J. Loos, R.A.J. Janssen, J. Am. Chem. Soc., 130 (2008) 7721-7735.

[28] J.K.J. van Duren, X.N. Yang, J. Loos, C.W.T. Bulle-Lieuwma, A.B. Sieval, J.C. Hummelen, R.A.J. Janssen, Adv. Funct. Mater., 14 (2004) 425-434.

[29] W. Li, L. Yang, J.R. Tumbleston, L. Yan, H. Ade, W. You, Adv. Mater., (2014) DOI: 10.1002/adma.201305251.

[30] A.J. Moule, J.B. Bonekamp, K. Meerholz, J. Appl. Phys., 100 (2006) 094503.

[31] C. Müller, T.A.M. Ferenczi, M. Campoy-Quiles, J.M. Frost, D.D.C. Bradley, P. Smith, N. Stingelin-Stutzmann, J. Nelson, Adv. Mater., 20 (2008) 3510-3515.

[32] D. Qian, W. Ma, Z. Li, X. Guo, S. Zhang, L. Ye, H. Ade, Z. Tan, J. Hou, J. Am. Chem. Soc., 135 (2013) 8464-8467. 
[33] X. Guo, M. Zhang, J. Tan, S. Zhang, L. Huo, W. Hu, Y. Li, J. Hou, Adv. Mater., 24 (2012) 6536-6541.

[34] F.C. Jamieson, E.B. Domingo, T. McCarthy-Ward, M. Heeney, N. Stingelin, J.R. Durrant, Chem. Sci., 3 (2012) 485-492.

[35] B.M. Savoie, A. Rao, A.A. Bakulin, S. Gelinas, B. Movaghar, R.H. Friend, T.J. Marks, M.A. Ratner, J. Am. Chem. Soc., 136 (2014) 2876-2884.

[36] P.A. Troshin, H. Hoppe, J. Renz, M. Egginger, J.Y. Mayorova, A.E. Goryachev, A.S. Peregudov, R.N. Lyubovskaya, G. Gobsch, N.S. Sariciftci, V.F. Razumov, Adv. Funct. Mater., 19 (2009) 779-788. 